\title{
Vulnerabilidad hidrogeológica en la zona costera de Jacó, Pacífico Central, Costa Rica
}

\author{
Hydrogeological vulnerability in Jacó coastal aquifers, \\ Central Pacific, Costa Rica
}

\author{
Alicia Gómez-Cruz ${ }^{1}$ \\ Helga Madrigal-Solis ${ }^{2}$ \\ Christian Núñez-Solis ${ }^{3}$ \\ Hazel Calderón-Sánchez ${ }^{4}$ \\ Pablo Jiménez-Gavilán ${ }^{5}$ \\ Universidad Nacional, Costa Rica
}

\begin{abstract}
Resumen
En las últimas dos décadas, el Pacífico Central de Costa Rica experimentó un fuerte aumento en el desarrollo urbanístico y turístico, lo que representa una mayor amenaza a la calidad del agua subterránea. El objetivo de este estudio fue el generar una propuesta del Mapa de Vulnerabilidad Hidrogeológica de los acuíferos de Jacó, utilizando la metodología GOD. Para esto, se elaboraron perfiles geológicos y se realizó comprobación de campo. Se evidencia que el área de estudio está conformada por a) un acuífero costero detrítico, poroso y libre, albergado en la planicie aluvial de Jacó y, b) un acuífero fracturado en las rocas basálticas del Complejo de Nicoya, libre en la zona montañosa y subyaciendo la formación detrítica en la zona central, donde el agua subterránea se integra a la del acuífero detrítico, conformándose en un mismo acuífero. La zona del acuífero

1 Licda. Alicia Gómez-Cruz. Laboratorio de Hidrología Ambiental. Escuela de Ciencias Biológicas. Universidad Nacional de Costa Rica. agomezcruz@gmail.com.

2 MSc. Helga Madrigal-Solís. Laboratorio de Hidrología Ambiental. Escuela de Ciencias Biológicas. Universidad Nacional de Costa Rica. helga.madrigal.solis@una.cr.

3 MSc. Christian Núñez-Solís. Laboratorio de Hidrología Ambiental. Escuela de Ciencias Biológicas. Universidad Nacional de Costa Rica. christian.nunez.solis@una.cr.

4 Licda. Hazel Calderón-Sánchez. Laboratorio de Hidrología Ambiental. Escuela de Ciencias Biológicas. Universidad Nacional de Costa Rica. hazelcalderon@gmail.com.

5 Dr. Pablo Jiménez-Gavilán. Departamento de Ecología y Geología. Facultad de Ciencias. Universidad de Málaga.pgavilan@uma.es.
\end{abstract}


aluvial obtuvo una vulnerabilidad moderada, en tanto que el fracturado una vulnerabilidad baja. Se propusieron recomendaciones de manejo.

Palabras clave: acuífero costero, vulnerabilidad hidrogeológica, GOD, acuífero fracturado, protección del agua subterránea.

\begin{abstract}
In the last two decades, the Central Pacific coast of Costa Rica experienced a strong growth in urban and touristic development. If not regulated, this development can result in a pressure to groundwater resources and, therefore, a threat in terms of quality and quantity. In this study, a hydrogeological vulnerability map of Jacó aquifers was performed, by the GOD method. Geological profiles were elaborated, and field verification was performed. It is confirmed that, in the study area, two aquifers are found: a) a detritic, porous, unconfined coastal aquifer, settled in the Jacó alluvial plain and, b) an aquifer in the basaltic fractured rocks of the Nicoya Complex, unconfined in the mountainous zone and underlying the detritic formation in the central area, where the groundwater is integrated to that of the detrital aquifer. The zone of the alluvial aquifer resulted in a medium vulnerability index, whereas the mountainous areas in a low vulnerability. Management recommendations were proposed.
\end{abstract}

Keywords: coastal aquifer, hydrogeological vulnerability, GOD index, fractured aquifer, groundwater protection.

\title{
Introducción
}

A nivel mundial, el acceso al agua potable se ha visto amenazado debido a un aumento en la demanda para satisfacer una mayor cantidad y variedad de actividades antropogénicas, una carga contaminante mayor y un uso no racional del recurso (Llamas y Custodio, 2003). Con base en los modelos de desarrollo (Foster, 1992), el Banco Mundial (BM) ha recomendado la elaboración de las cartografías de amenaza, vulnerabilidad y riesgo de los acuíferos a la contaminación como una medida para la protección de la calidad de las aguas subterráneas (Foster et al., 2007). El concepto de vulnerabilidad se remonta desde la década de los 70 (Albinet y Margat, 1970), de forma intuitiva; posteriormente, su uso fue ampliamente utilizado en los años 80 (Foster e Hirata 1988). De acuerdo con Foster et al. (2007), el concepto de vulnerabilidad del agua subterránea está en función de las características propias de los estratos que separan la zona no saturada de la saturada. Estas características determinan la tendencia o probabilidad de que algún contaminante alcance una posición especifica en el sistema acuífero, después de su introducción en algún punto sobre el terreno.

En Costa Rica, durante las últimas décadas, han surgido y se han consolidado políticas ambientales orientadas a reforzar la protección y uso 
Alicia Gómez-Cruz - Helga Madrigal-Solis - Christian Núñez-Solís

Hazel Calderón-Sánchez - Pablo Jiménez-Gavilán

Vulnerabilidad hidrogeológica en la zona costera de Jacó, Pacífico Central, Costa Rica.

racional de los recursos naturales. No obstante, la gestión del recurso hídrico enfrenta grandes desafíos, los cuales deben superarse con acciones eficientes para garantizar la calidad ambiental y la sostenibilidad del recurso hídrico subterráneo (Reynolds y Fraile, 2002). Por tal motivo, a partir del 2008, el Estado dictaminó la elaboración de estudios de vulnerabilidad hidrogeológica para ser incluidos en la normativa urbanística de cada cantón del país, según el método GOD (Groundwater Hidraulic Confinement, Overlaying Strata and Deph to Groundwater Table). El método GOD considera dos factores básicos: el grado de inaccesibilidad hidráulica de la zona saturada y la capacidad de atenuación de los estratos suprayacentes a la zona saturada (Foster e Hirata, 1991). Esta metodología utiliza los siguientes parámetros (Foster et al. 2007): grado de confinamiento hidráulico $(\mathrm{G})$, características de los sustratos de la zona no saturada o capas confinantes, tomando en cuenta aspectos litológicos y el grado de consolidación $(\mathrm{O}) \mathrm{y}$; distancia al agua, considerada como la profundidad al nivel freático o al techo del acuífero confinado (D). Se entiende por grado de confinamiento hidráulico si el acuífero es libre, confinado o semiconfinado (Foster et al. 2007).

El abastecimiento de agua potable ha sido un factor clave para el desarrollo de las comunidades costeras. Sin embargo, el crecimiento desordenado en la zona marítimo-terrestre en el litoral pacífico de Costa Rica pone en evidencia el seguimiento limitado a las regulaciones existentes (Estado de la Nación, 2007). Además, en los últimos años, se ha generado un rápido crecimiento urbano y turístico en esta zona (Barrantes, 2004). Según el Instituto Nacional de Estadísticas y Censos (INEC, 2012), el cantón de Garabito, al cual pertenece la comunidad de Jacó, ha presentado la mayor tasa de crecimiento poblacional del país durante los últimos 30 años, lo que podría conllevar a un aumento en la carga de contaminantes que se liberan al ambiente (Orozco, 2015).

Por su parte, lo somero del nivel freático, en algunos sectores (Arias y Morera, 2002), implica que las actividades antrópicas representen una amenaza a la calidad dael agua subterránea. Debido al rápido crecimiento urbano y turístico de la ciudad de Jacó, es necesario contar con una herramienta que contribuya con el ordenamiento territorial para la protección de los recursos hídricos subterráneos, los cuales son ampliamente utilizados para consumo humano y otras actividades productivas. Por esta razón, el 
objetivo del presente estudio es elaborar una propuesta de Mapa de Vulnerabilidad Hidrogeológica $(\mathrm{MVH})$ en este sector.

\section{Área de estudio}

El sitio de estudio (Figura 1) está localizado en el distrito de Jacó, cantón de Garabito de Puntarenas, Costa Rica, entre las coordenadas geográficas $9^{\circ} 35^{\prime} 40,66^{\prime \prime}$ - 940'34,647" latitud norte y 84³8'48,466" $84^{\circ} 34^{\prime} 27,373^{\prime \prime}$ longitud oeste, y entre las coordenadas $1061000 \mathrm{~m}-1$ 070000 m norte y 429000 m - 437000 m este, del sistema de Proyección Transversal de Mercator para Costa Rica (CRTM). La zona de estudio está delimitada por una cuenca hidrográfica de nombre Jacó, nombre que ha sido asignado en este estudio para el área en análisis, con una extensión de $47 \mathrm{~km}^{2}$; compuesta por tres microcuencas que pertenecen a los ríos Pueblo Nuevo (Mona), Copey y Naranjal. En la parte baja y media de estas microcuencas se localiza un acuífero albergado en depósitos no consolidados, con un área aproximada de $15 \mathrm{~km}^{2}$. El área restante está compuesta, principalmente, por basaltos y rocas sedimentarias, en donde se encuentra un acuífero fracturado. Estas rocas se ubican en los cerros (Figura 2).

Figura 1. Mapa de ubicación de la zona de estudio, Jacó, Puntarenas, Costa Rica.
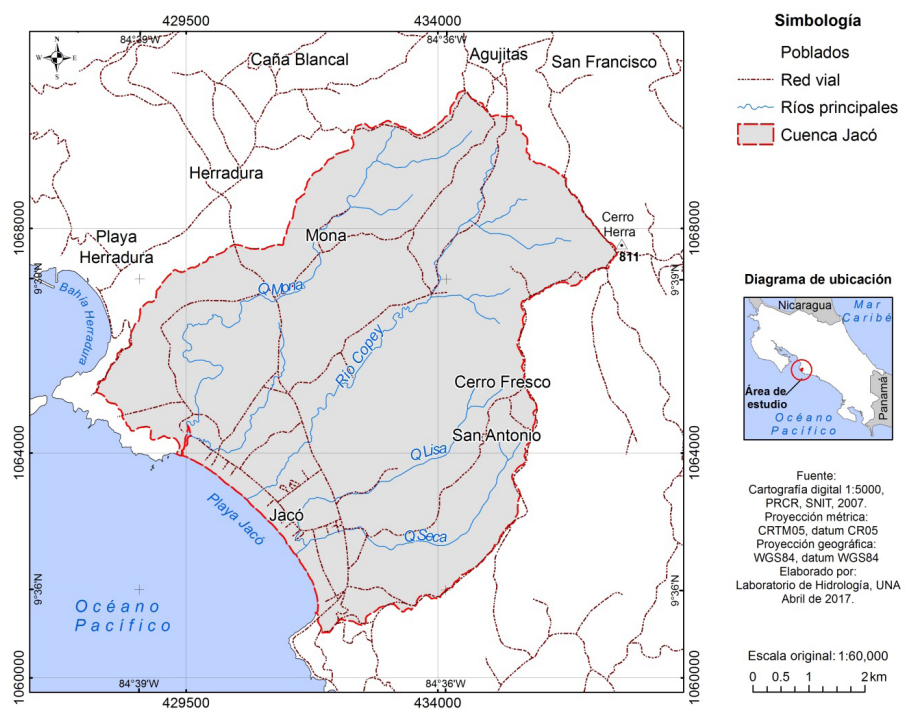
Figura 2. Mapa geológico de la zona de estudio, Jacó, Puntarenas, Costa Rica. Modificado de Arias (2003). Redefinición de la Formación Tulín (Maastrichtiano-Eoceno Inferior) del Pacífico Central de Costa Rica.

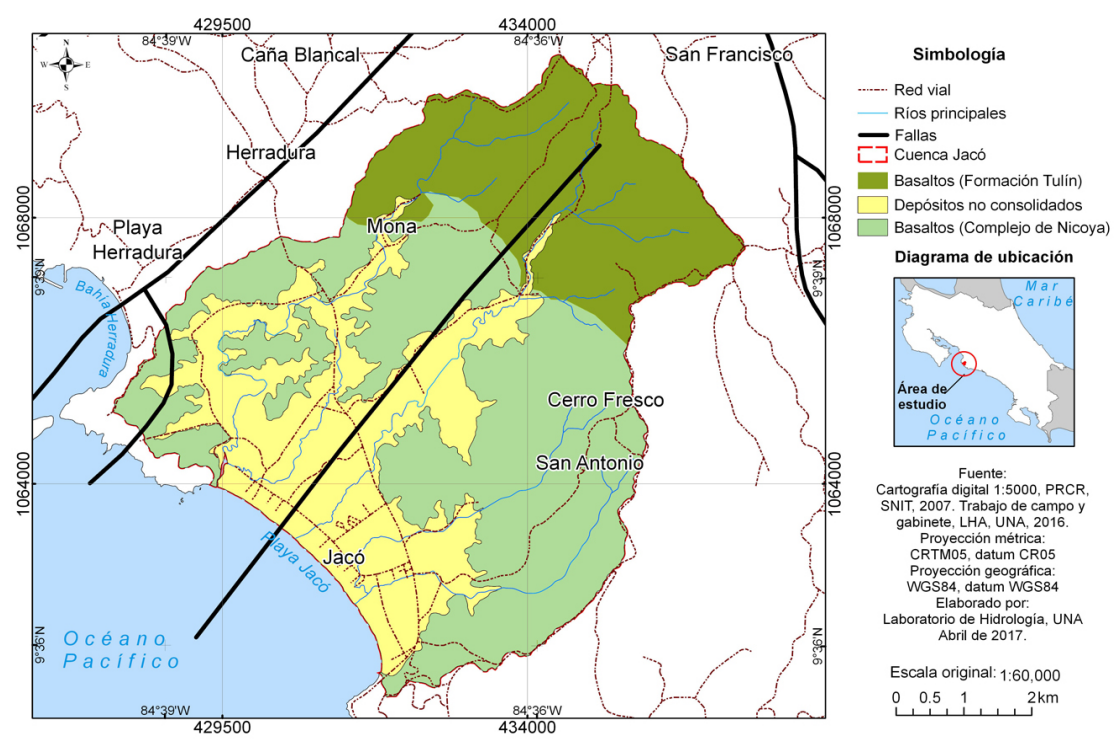

\section{Características generales}

La época seca se extiende por cinco meses, comprendidos de diciembre a abril, siendo enero el más seco, en tanto que la época lluviosa comprende los meses de mayo a noviembre y el mes más lluvioso corresponde con octubre. Según la base de datos del Instituto Meteorológico Nacional de Costa Rica del 2014, en la Estación Jacó, la precipitación máxima promedio, entre 1997 y 2013, fue de 616,6 mm en los meses de octubre; el mínimo de precipitación se obtuvo en los meses de enero, con un promedio de $25 \mathrm{~mm}$.

\section{Aspectos geológicos}

El área de estudio está conformada por tres tipos de unidades litoestatigráficas claramente diferenciables entre sí: a) basaltos del Complejo de Nicoya, dentro del cual se han incluido rocas tipo radiolaritas, calizas, 
areniscas y lutitas, b) basaltos asociados a la Formación Tulín y, c) depósitos no consolidados (Figura 2).

Complejo de Nicoya. Dengo (1962) define el Complejo de Nicoya como una serie de rocas intensamente plegadas, consistentes en basaltos, unidades sedimentarias e intrusivas. Kuypers (1980, citado en Sprechmann et al., 1984) indica que existe ambigüedad debido a que las series de rocas suprayacentes se encuentran, localmente, muy plegadas y a la presencia de extrusiones basálticas. Por lo tanto, se incluyen diferentes litologías dentro de la unidad y, en consecuencia, varía la edad que se le atribuye. Debido a lo anterior, Kuypers (1980, citado en Sprechmann et al., 1984) y Baumgartner (1984) proponen una subdivisión estratigráfica para el Complejo de Nicoya, la que consiste en dos unidades, Matapalo y Esperanza. Arias (2003) denomina a los basaltos que han sido asociados al Complejo de Nicoya como basaltos del Plateau Oceánico Caribe (POC); estos basaltos son las rocas más antiguas.

La Unidad Matapalo posee $150 \mathrm{~m}$ de espeso; se caracteriza por estar conformada por basaltos tholeíticos oceánicos cubiertos por radiolaritas, con espesor mínimo de $40 \mathrm{~m}$. La edad asignada es del Calloviense hasta el Aptiense (Kuypers, 1980, citado en Sprechmann et al., 1984). El contacto con la suprayacente Esperanza no es claramente visible (Kuypers, 1980, citado en Sprechmann et al., 1984). En la Unidad Esperanza, conformada por basaltos tholeiíticos y diabasas ofiolíticas, son comunes las rocas gabroicas, stocks plagiograníticos y algunas intercalaciones de radiolaritas de hasta $3 \mathrm{~m}$ en la parte superior de la unidad. La edad asignada es del Santoniense (Kuypers, 1980, citado en Sprechmann et al., 1984).

En la zona de estudio, la geología en los cerros está compuesta de basaltos y rocas sedimentarias tipo areniscas, lutitas, radiolaritas y calizas que se asocian a las rocas del Complejo de Nicoya (Arias y Morera, 2002). Estas lavas, de composición basáltica, son de una coloración negra y, por su origen, tienen una textura en almohadilla, lo que las caracteriza. En superficie, están meteorizadas y son fácilmente confundibles con arenas gruesas.

Las radiolaritas del Complejo de Nicoya forman parte de la Unidad Matapalo, del Jurásico Superior - Cretácico Inferior. Son rocas de origen pelágico, de grano fino a muy fino, estratificadas, bastante duras y versicolor (Agudelo, 2005). Están relacionadas a pedernales y jaspes 
y se encuentran muy a menudo asociadas a mineralizaciones de hierromanganeso, resultado de la alteración hidrotermal en fondos oceánicos a partir de vulcanismo exhalativo, sea en forma diseminada o en nódulos. Las radiolaritas sobreyacen disconformemente la unidad de basaltos y es atravesada por numerosos cuerpos intrusivos gabroides a diabásicos. Presenta una fuerte deformación tectónica (pliegues isoclinales-chevron) y un alto grado de fallamiento (Agudelo, 2005).

Formación Tulín. Arias (2003) sugiere que la Formación Tulín, del (Maastrichtiano-Eoceno Inferior, está conformada en un $95 \%$ por basaltos, los cuales se presentan como coladas en almohadillas; estos basaltos presentan intercalaciones de sedimentos epiclásicos, piroclasto, gabros y cumulitas de olivino. Es una formación más joven que el POC, cuyas rocas constituyen el basamento de la Formación Tulín.

Depósitos no consolidados. Pertenecen a esta unidad todos los depósitos recientes asociados a los depósitos por gravedad al pie de monte o coluvios y a aquellos relacionados con procesos aluviales. También aquellos depósitos de materiales arenosos con influencia marina. Los depósitos asociados al arrastre por acción fluvial o por gravedad, por lo general, son fragmentos de rocas de diversos tamaños y composición inmersos en una matriz limo-arcillo arenosa. Hacia el pie de los cerros, los bloques de fragmentos son de mayor tamaño y sub-redondeados a redondeados, en tanto que hacia la parte baja de la cuenca los fragmentos son más pequeños, ya que han sido arrastrados a mayor distancia, lo que se evidencia por su menor grado de angulosidad (Arias y Morera, 2002).

\section{Marco teórico-conceptual}

La estimación del índice de vulnerabilidad GOD (Foster e Hirata, 1988) involucra una serie de etapas concretas. Primera, identificar el grado de confinamiento hidráulico del acuífero y asignarle un índice a este parámetro en una escala de 0.0 a 1.0. Segunda, especificar las características del sustrato suprayacente a la zona saturada del acuífero en términos de: a) grado de consolidación (teniendo en cuenta la probable presencia o ausencia de permeabilidad por fisuras) y, b) tipo de litología (considerando indirectamente porosidad efectiva, permeabilidad de la matriz y contenido de humedad en la zona no saturada o retención específica) y, asignar un índice a este parámetro en una escala de 0.4 a 1.0. Tercera, estimar la distancia o 
profundidad al nivel del agua (en acuíferos no confinados) o profundidad al techo del primer acuífero confinado, con la consiguiente asignación de un índice en una escala de 0.6 a 1.0. En este sentido, el peligro de contaminación del agua subterránea se definiría luego como la probabilidad de que el agua subterránea, en la parte superior de un acuífero, sea contaminada en un nivel inaceptable por las actividades que se desarrollan en la superficie del terreno suprayacente (Foster e Hirata, 1988; Foster et al. 2007).

Así, en lugar de aplicar controles universales sobre los usos del territorio potencialmente contaminantes y la descarga de efluentes, es más efectivo (y menos perjudicial para el desarrollo económico) modificar el tipo y nivel de control de acuerdo con esta capacidad de atenuación. Esta es la premisa básica detrás del concepto de vulnerabilidad a la contaminación de acuíferos y lo que conduce a la necesidad de contar con el mapeo de la misma (Foster et al., 2007).

\section{Marco metodológico}

Perfiles geológicos. En la zona de estudio existen registrados 57 pozos privados y cuatro pozos de abastecimiento público pertenecientes al AyA. De estos 57, solamente 21 cuentan con la descripción litológica en sus informes de perforación. Estos informes se recuperaron en octubre del 2016 del archivo institucional de la Dirección de Investigación y Gestión Hídrica del Servicio Nacional de Aguas Subterráneas, Riego y Avenamiento (SENARA) y del Archivo de Concesiones de la Dirección de Aguas del Ministerio de Ambiente y Energía (MINAE). Con base en esta información, se trazaron al menos 11 perfiles geológicos, de los que se escogieron los cuatro perfiles más representativos para determinar la relación entre las unidades geológicas. Un perfil se trazó con una dirección de suroeste a noreste, el segundo perfil de noroeste- sureste, atravesando el sector de la cuenca baja y el tercero de noroeste-sureste, atravesando el sector de la cuenca alta.

Aforos diferenciales. Para definir la relación hidráulica entre las aguas superficiales y las subterráneas se planificaron dos campañas de aforos diferenciales, una en época seca y otra en la época lluviosa, en los principales ríos y quebradas de la cuenca. Los aforos diferenciales han sido ampliamente utilizados en diversas situaciones climáticas para realizar 
estimaciones de caudal, por ejemplo, en Andreo et al. (2004) y Barberá y Andreo (2015). Para la selección de los sitios se consideró que éstos no tuvieran influencia por entradas o salidas de aguas provenientes de otros cursos de agua, y si las había, se aforaban estos ingresos o egresos de agua para valorar la relación hidráulica entre el río y el acuífero. En noviembre del 2013, durante la época lluviosa, se realizaron aforos en 18 sitios; en marzo del 2014, época seca, en 12 sitios. La diferencia entre ambas campañas se debe a que seis de los puntos aforados en época lluviosa se encontraban secos para la época de estiaje. Una vez calculado el caudal en cada punto aforado, se definieron los tramos donde el río es influente y los tramos en donde el río es efluente.

Mapa de vulnerabilidad. Se utilizó el método GOD (Foster et al., 2002), para caracterizar la vulnerabilidad de los acuíferos en la zona de estudio. Para la obtención de los valores de vulnerabilidad en cada una de las capas de las que se compone el método GOD, se hizo una revisión del Mapa Geológico de Costa Rica (Denyer y Alvarado, 2007), del Atlas tectónico de Costa Rica (Denyer et al., 2003), del estudio de Arias (2003) y de la Cartografía digital y ortofoto (PRCR, 2007). Además, se hizo corroboración de campo de la geología de la zona, correlacionando los afloramientos observados con las descripciones realizadas por los autores mencionados. También, se recopiló la información litológica de los pozos en la zona a partir de los informes de perforación archivados en el SENARA (Servicio Nacional de Aguas Subterráneas Riego y Avenamiento) y en la Dirección de Aguas del Ministerio de Ambiente y Energía, y de los datos de perforación de los pozos del AyA, para estimar el espesor de la zona no saturada y la profundidad del techo de los acuíferos. Finalmente, se utilizaron los resultados de los perfiles geológicos elaborados durante esta investigación para mejorar el entendimiento del modelo hidrogeológico conceptual. Se utilizó un Sistema de Información Geográfica (SIG) para integrar la información requerida para la estimación de los valores en cada capa del método GOD. Una vez definidas las vulnerabilidades en el área de estudio, se elaboró una matriz con las actividades sugeridas en cada zona, según su vulnerabilidad, considerando las actividades desarrolladas en la región. 


\section{Resultados \\ - Hidrometría}

Durante las campañas de medición de caudal, se corroboró en el campo la ausencia de tomas de agua para riego, embalses o cualquier otro aprovechamiento a lo largo del río o quebrada. De esta forma, se confirmó el carácter influente-efluente de los ríos estudiados. La quebrada Mona, en época lluviosa, tuvo un tramo en el sector central de su recorrido en donde el caudal pasó de 79,9 a 14,7 1/s. Esta reducción en el caudal indica que en este sector la quebrada se comportó como influente y el tramo que sigue hasta su desembocadura como efluente (Figura 3). El río Copey, en época lluviosa, se comportó como efluente en la mayor parte del trayecto, excepto el sector medio, en donde el caudal pasó de 133,2 a 112,9 1/s. La quebrada Seca, también en época lluviosa, tuvo un comportamiento efluente, excepto en su desembocadura, donde su caudal cambió de 99,6 a 52,4 1/s, convirtiéndose en una quebrada influente en ese sitio (Figura 3). En época de lluvia, el comportamiento influente-efluente de los ríos a lo largo de su cauce evidenció que el acuífero tiene una profundidad al nivel de agua subterránea por encima del lecho del río, de tal manera que el acuífero puede alimentar los ríos y quebradas, mientras que en otros sectores la posición del nivel del agua está por debajo del lecho del río, invirtiendo el comportamiento. En época seca, el comportamiento de los ríos se mantuvo igual, y lo único que varió fue el caudal. Así mismo, los tramos en los que el caudal disminuyó drásticamente en época lluviosa son los mismos que se encontraron completamente secos en época seca debido al descenso del nivel del agua en el acuífero (Figura 3), lo que corrobora el carácter influente de los ríos y quebradas en estos sectores.

\section{- Modelo hidrogeológico}

En la zona de Jacó se han desarrollado dos acuíferos costeros; uno superficial, detrítico, libre, albergado en el depósito no consolidado y, otro fracturado y albergado en las rocas del Complejo de Nicoya (Figura 4). Este acuífero fracturado es libre y profundo en los cerros y, cuando subyace al acuífero detrítico en la llanura aluvial se puede mostrar confinado en al menos un sector de la zona central de la llanura, donde la carga de presión alcanza hasta $1 \mathrm{~m}$ por encima del nivel del terreno (registros de 
Figura 3. Mapa de aforos diferenciales en la zona de estudio, Jacó, Puntarenas, Costa Rica.
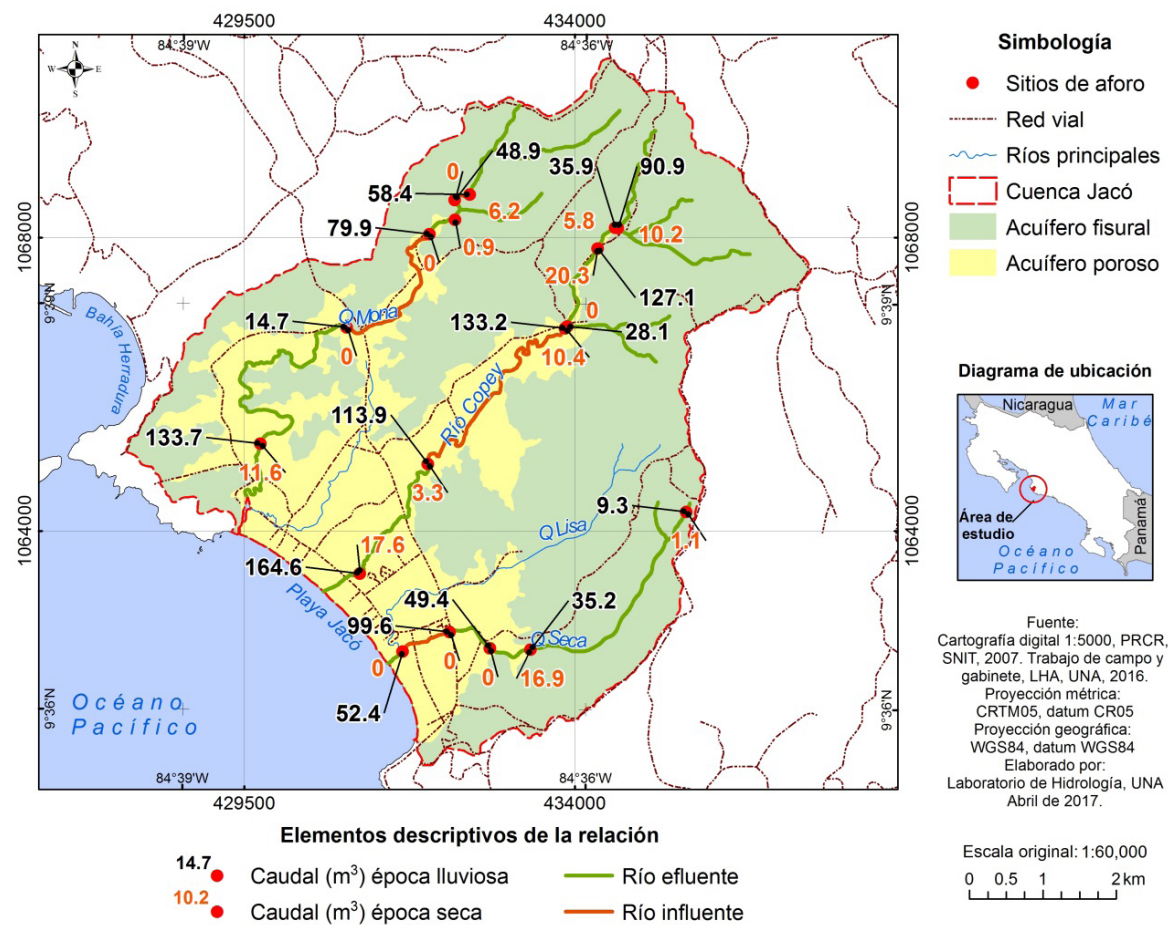

Diagrama de ubicación

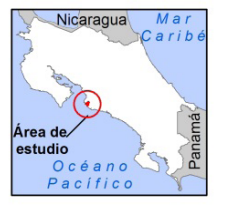

Fuente:

Cartografia digital 1:5000, PRCR SNIT, 2007. Trabajo de campo y gabinete, LHA, UNA, 2016.

Proyección métrica: royección geográfica:

WGS84, datum WGS84 Elaborado por: Laboratorio de Hidrologia, UNA Abril de 2017

Escala original: $1: 60,000$ $0.5,1,2 \mathrm{~km}$

perforación HE-134 y Pozo 5 del AyA) (perfil 4, Figura 6). El agua subterránea en las zonas montañosas viaja a través de los basaltos y rocas sedimentarias; luego, probablemente, una parte se integra al agua subterránea del depósito no consolidado y conforma un único acuífero (perfiles 1 y 2, Figuras 5 y 6). Aun cuando este acuífero estuviera conformado por dos formaciones geológicas, materiales no consolidados y basaltos por debajo de ellos, con distintos valores de conductividad hidráulica y permeabilidad, es probable que el agua subterránea mantenga un mismo nivel por la ausencia de una capa impermeable que separe ambas formaciones, lo que se deduce a partir de los informes de perforación obtenidos del SENARA. En el informe del pozo HE-134 se indicó, que cuando el pozo alcanzó los basaltos fracturados, a los $22 \mathrm{~m}$ de profundidad, se produjo una surgencia 
(Figura 6, perfil 4). La surgencia reportada en ese informe y en el Pozo 5 del AyA podría deberse a: a) la diferencia en permeabilidad de la formación fracturada con respecto a la detrítica; b) a una mayor presión del agua en dicho material fracturado debido al efecto de algunos lentes de arcilla cerca de la superficie (Figura 6, perfil 4) o; c) los pozos que alcanzaron los basaltos están expuestos a un mayor potencial hidráulico, debido a que alcanzaron profundidades con distintas líneas equipotenciales a las de los pozos que extraen agua de la formación detrítica; sin que estas tres razones sean mutuamente excluyentes. Además, en los perfiles 1 y 2 (Figura 5) se evidencia que la topografía de los basaltos es muy irregular y el nivel del agua subterránea en estos basaltos es afectado por las fuertes pendientes.

Figura 4. Mapa hidrogeológico de la zona de estudio, Jacó, Puntarenas, Costa Rica.

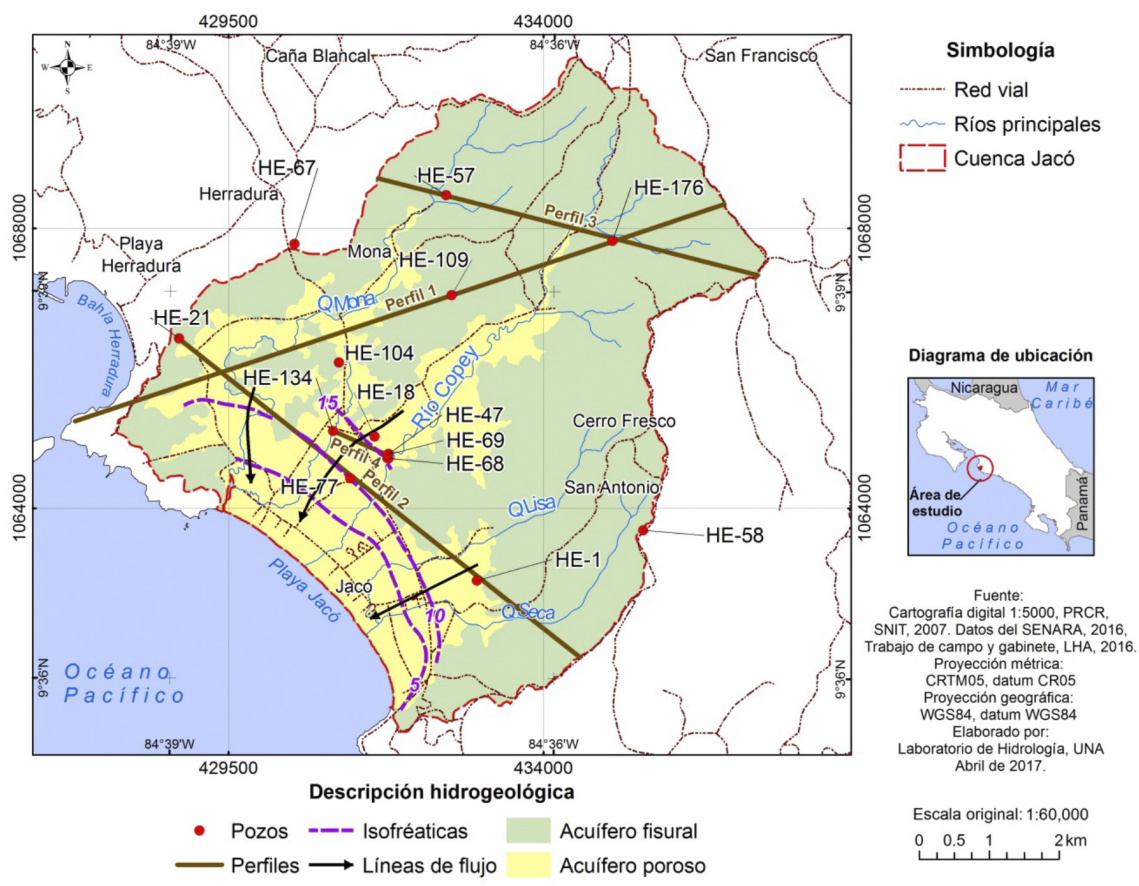


Figura 5. Perfiles hidrogeológicos 1 y 3 en la zona de estudio, Jacó, Puntarenas, Costa Rica.
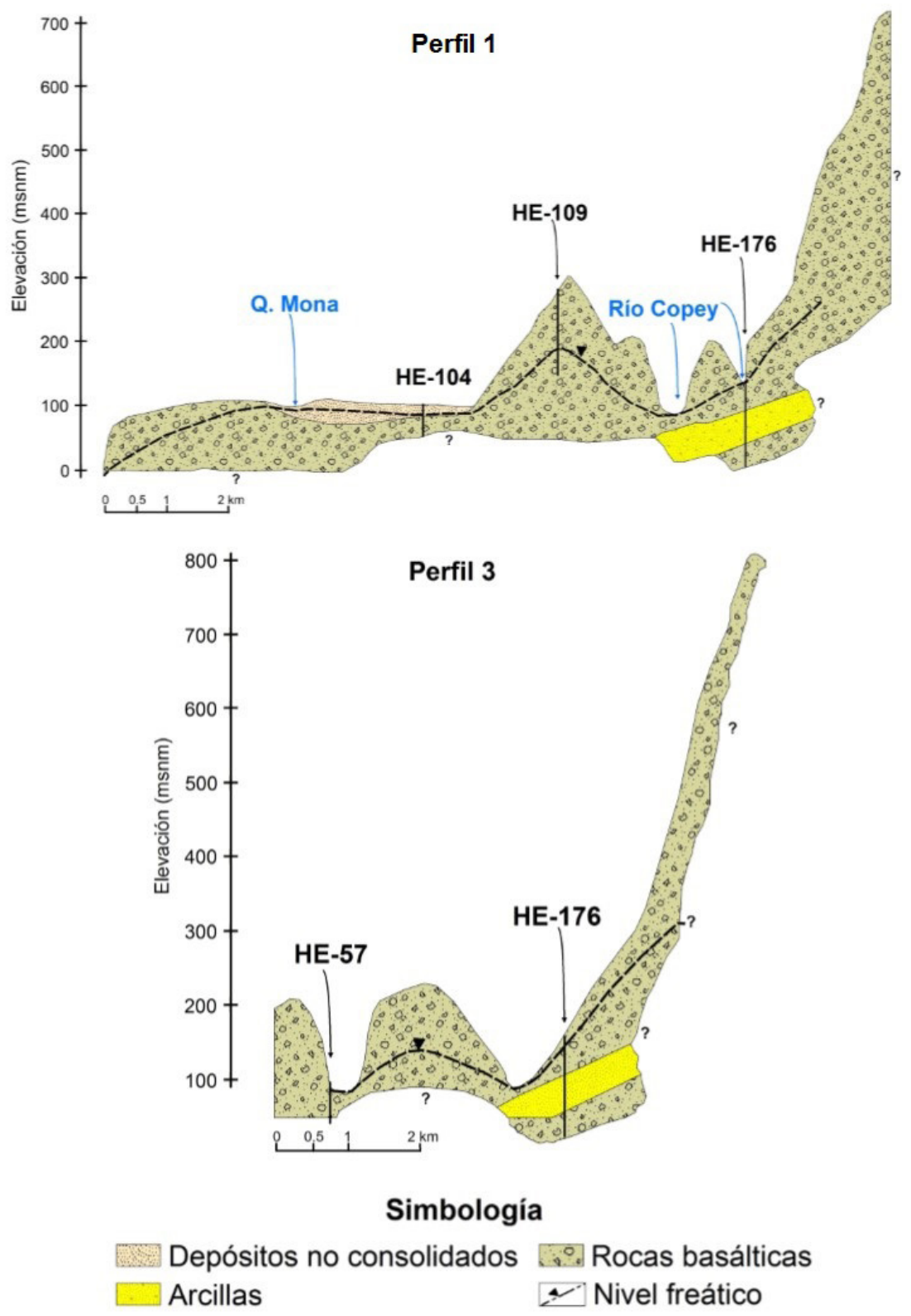
Figura 6. Perfiles hidrogeológicos 2 y 4 en la zona de estudio, Jacó, Puntarenas, Costa Rica.
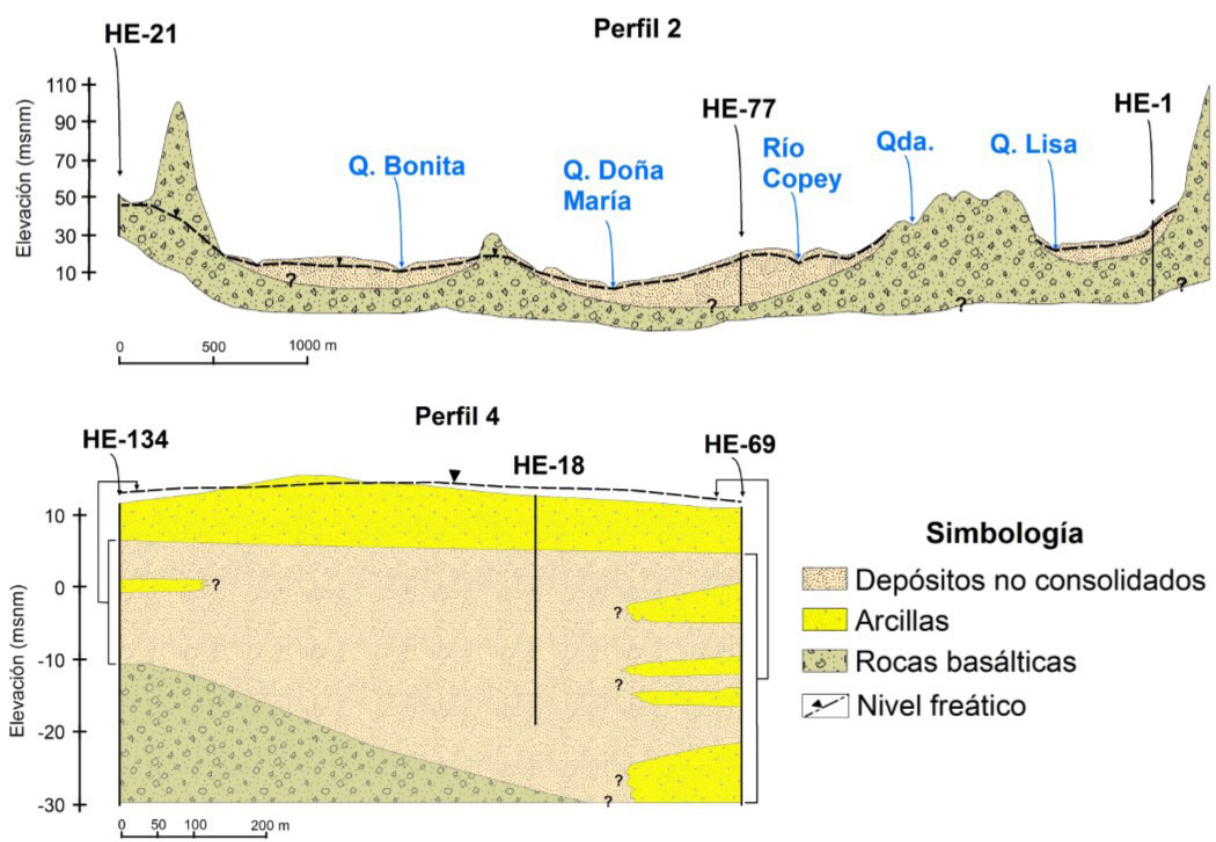

\section{- Acuífero detrítico Jacó.}

Se trata de un acuífero, libre, detrítico, albergado en materiales no consolidados tipo arenas, limos y gravas con una capa de suelo de poco espesor. En los sectores con información de nivel de agua, este se encuentra entre 0,5 y $5 \mathrm{~m}$ de profundidad, con equipotenciales entre los 0,5 y 15 m.s.n.m. (Figura 4). La dirección hidráulica de este acuífero es hacia el suroeste $(\mathrm{SW})$ y el gradiente es de 0,01 . Otro mecanismo de recarga se da por infiltración desde el lecho de los ríos, como se ha demostrado con los aforos diferenciales realizados (Figura 3). La descarga se produce de manera natural, como descarga a los ríos, en algunos sectores (Figura 3), y hacia al mar (Figura 4), y producto de la extracción de los pozos. En la planicie aluvial no se encontraron manantiales durante las visitas al campo ni tampoco existen registros. Los caudales de explotación indican una productividad variable desde 1 hasta 50 1/s. Los pozos que reportan un caudal de extracción mayor son los pozos que alcanzaron los basaltos fracturados. 
Alicia Gómez-Cruz - Helga Madrigal-Solis - Christian Núñez-Solís

Hazel Calderón-Sánchez - Pablo Jiménez-Gavilán

Vulnerabilidad hidrogeológica en la zona costera de Jacó, Pacífico Central, Costa Rica.

\section{- $\quad$ Acuífero fracturado.}

Este acuífero fracturado, con una permeabilidad secundaria originada por las fisuras, se ha desarrollado en lavas y rocas sedimentarias mencionadas anteriormente, las que conforman los cerros que rodean la cuenca. En este acuífero fracturado, existían 19 pozos registrados hasta la fecha; únicamente 15 con información litológica. La profundidad del nivel del agua subterránea es variable debido a que los pozos tienen profundidades variables y el nivel del agua resultante corresponde con un nivel de equilibrio entre las distintas capas productoras. Por ejemplo, el pozo HE-109 posee $105 \mathrm{~m}$ de profundidad y un nivel estático reportado a $70 \mathrm{~m}$ de profundidad, en tanto que el pozo HE-58 tiene $180 \mathrm{~m}$ de profundidad y un nivel estático a $100 \mathrm{~m}$ de profundidad. En los informes de los pozos HE-18, HE-19, HE-47, HE-68 y HE-134, en la zona central, se reportó surgencia entre los 22 y los 38 m, en donde se ubican los basaltos fracturados (perfil 4, Figura 6).

En cuanto a la productividad del acuífero, los informes de perforación obtenidos de los archivos del SENARA indican caudales muy bajos, entre 0,5 a $71 / \mathrm{s}$, con descensos muy fuertes. A pesar de no contarse con pruebas de bombeo, esta condición, posiblemente, refleja un acuífero de baja productividad debido a una baja permeabilidad secundaria. Aun cuando no se pudo trazar equipotenciales para este acuífero por falta de información, es probable que el flujo se dirija hacia las zonas aluviales, ya que las rocas que contienen este acuífero se encuentran en los cerros bordeando la llanura. Posteriormente, en el sector subyacente al acuífero detrítico, el agua se integra con el agua subterránea en el acuífero detrítico, conformándose un único nivel y un único acuífero. Luego, el flujo se dirige hacia el SW, es decir, hacia el mar. Sin embargo, esta es una suposición basada en las características topográficas y geomorfológicas de la zona (Figura 4).

\section{- Vulnerabilidad hidrogeológica}

Una vez definidas las condiciones hidrogeológicas, se estimó la vulnerabilidad del acuífero poroso y del acuífero fracturado en la zona de los cerros.

Grado de confinamiento hidráulico (Valor $G$ ). Al acuífero detrítico se le asignó un valor de 0,9 , por ser un acuífero con poca cobertura, es decir, con una zona vadosa de poco espesor, según lo observado mediante los perfiles realizados. Para el acuífero fracturado se estimó un valor de 0,7, correspondiente a un acuífero libre con cobertura de acuerdo a los perfiles 


\section{1, 2 y 3. (Figura 7). Ocurrencia del sustrato suprayacente (Valor O). Con} base en la descripción litológica en los reportes de perforación de los pozos, se identificó una capa de suelo arcillo-limoso en la zona del acuífero aluvial, de espesor variable entre los 0 y $14 \mathrm{~m}$, por lo que se estimó un valor de 0,5. En los cerros, el valor es de 0,6, corresponde a rocas basálticas fracturadas (Figura 7). Distancia al nivel del agua subterránea (Valor D). En el acuífero detrítico, la profundidad del nivel de agua con respecto al nivel de suelo se encuentra entre 1 y $5 \mathrm{~m}$, por lo que se le asignó un valor de 0.9 , mientras que, en el acuífero libre fracturado, en la zona de los cerros, el agua se encontró entre 20 y $50 \mathrm{~m}$ de profundidad, correspondiéndole un valor de 0,7 , aunque hay niveles en algunos pozos a mayor profundidad (Figura 7).

Figura 7. Grado de confinamiento (G), Ocurrencia del sustrato (O) y Distancia del agua (D) de los acuíferos en la zona de estudio, Jacó, Puntarenas, Costa Rica.

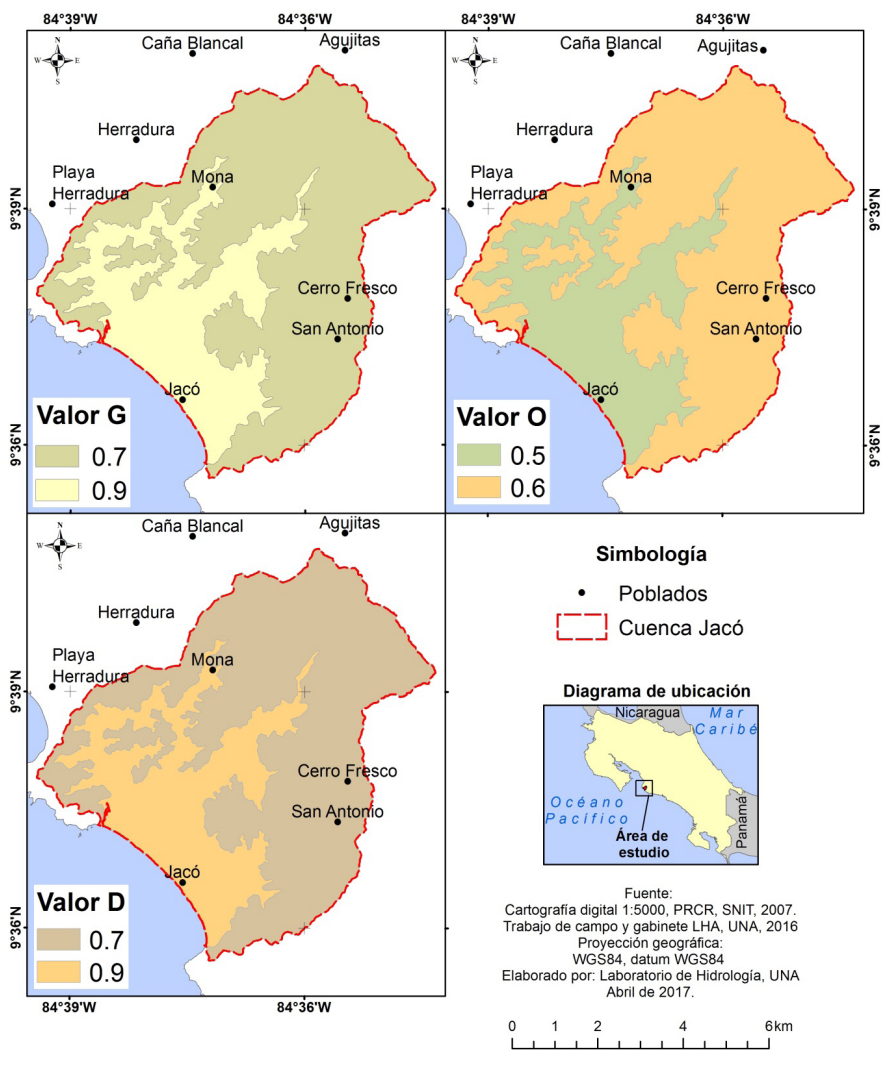


Como resultado, se obtuvo un nivel medio de vulnerabilidad hidrogeológica en la zona en donde se ubica el acuífero detrítico, con un valor de 0,41 (Figura 8). Este sector representó un 30,5 \% del total del área de estudio, mientras que el área correspondiente del acuífero fracturado se clasificó con un nivel bajo de vulnerabilidad hidrogeológica, con un valor de 0,29 .

Figura 8. Propuesta de Vulnerabilidad Hidrogeológica de los acuíferos en la zona de estudio, Jacó, Puntarenas, Costa Rica.
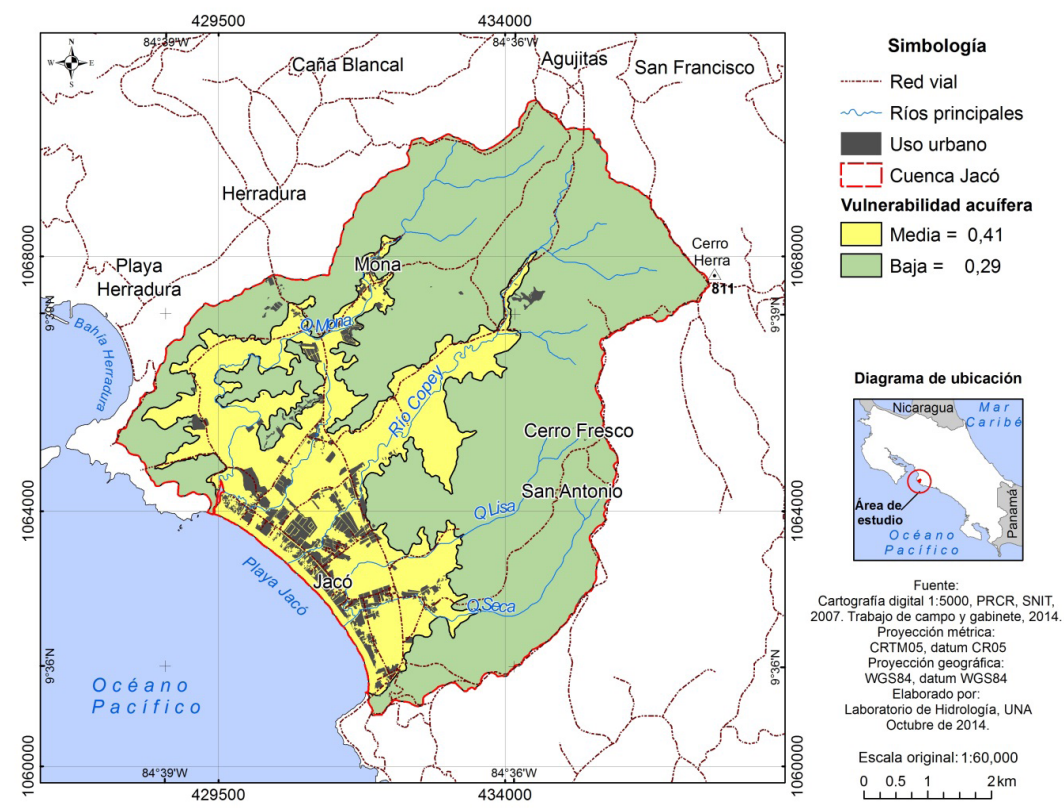

\section{Discusión}

Si bien la vulnerabilidad a la contaminación depende de la capacidad de atenuación natural del perfil de suelo a los contaminantes, esta varía ampliamente con las condiciones geológicas cercanas a la superficie del terreno. Entre estas condiciones se encuentra la profundidad a la que está el agua subterránea. Esto tuvo implicaciones importantes al designarle una vulnerabilidad baja al acuífero fracturado, ubicado en los cerros de la zona de Jacó, debido a que el nivel del agua se encontró a profundidades 
superiores a los $50 \mathrm{~m}$. Los acuíferos con vulnerabilidad baja son vulnerables a contaminantes conservativos cuando son descargados o lixiviados a largo plazo, con regularidad (Foster et al., 2007). Según Foster et al. (2013), un acuífero con vulnerabilidad baja puede ser vulnerable a la lixiviación de nitratos, líquidos altamente salinos y compuestos organoclorados en fase densa no acuosa.

Por su parte, aun cuando el nivel del agua subterránea es somero, el acuífero sobre la llanura aluvial obtuvo una clasificación de vulnerabilidad a la contaminación moderada. Esto debido a que los informes de los pozos en esta zona indican una capa de arcillas de varios metros de espesor en superficie, e inclusive indican intercalaciones de arcillas con las gravas dentro del paquete aluvial, lo que le ha dado a este acuífero cierto grado de protección ante ciertas sustancias contaminantes. Según Foster et. al. (2007), los acuíferos con vulnerabilidad moderada son aquellos que son vulnerables a algunos contaminantes cuando son descargados o lixiviados con regularidad. Aun cuando hay un pequeño sector dentro del acuífero en donde se reporta surgencia, los informes de los pozos no señalan la profundidad a la que ocurrió esta condición y los niveles de agua subterránea indicados en dichos informes corresponden con niveles de equilibrio entre el sector confinado y el acuífero detrítico más superficial. Por tal motivo, este sector se clasificó bajo la misma clase de vulnerabilidad que el resto del acuífero detrítico.

La inclusión de la vulnerabilidad hidrogeológica en el Plan Regulador de los gobiernos locales en Costa Rica resultaría de gran provecho para definir las actividades a desarrollar dentro del territorio municipal, lo que permitiría implementar procesos de gestión integrada del recurso hídrico. Para esto, la implementación de la propuesta del mapa de vulnerabilidad debe ir asociada a la aplicación de una matriz de vulnerabilidad. En el caso de Jacó, la mayor parte de los proyectos urbanísticos se ubican en el sector de vulnerabilidad moderada, así como las principales actividades productivas y socioeconómicas del área de estudio. Sin embargo, el plan regulador para la zona costera de Jacó, el cual no ha sido aprobado a la fecha, no contempla aún la vulnerabilidad del acuífero y permite un área de construcción por predio del $70 \%$, no siendo necesariamente la medida adecuada para un sector con vulnerabilidad moderada, tanto por razones de recarga como de carga hidráulica potencialmente contaminante. Tampoco 
Alicia Gómez-Cruz - Helga Madrigal-Solis - Christian Núñez-Solís

Hazel Calderón-Sánchez - Pablo Jiménez-Gavilán

Vulnerabilidad hidrogeológica en la zona costera de Jacó, Pacifico Central, Costa Rica.

existe una normativa que regule el tamaño de los predios a futuro en zonas no urbanizadas dentro del distrito. Además, se debe evaluar qué cantidad de predios actuales cumple la norma del 70 \% y cuáles serán las medidas de control a futuro.

La elaboración de los mapas de vulnerabilidad en la zona evidenció una necesidad de mayor inversión por parte de las instituciones gubernamentales para generar datos hidrogeológicos, principalmente a través de la instalación de más piezómetros en las áreas del Complejo de Nicoya. Esta indicación es necesaria para cualquier método de evaluación de la vulnerabilidad hidrogeológica (Foster et al., 2013). Debido a la escasez de información hidrogeológica en la zona de estudio, se debió extrapolar parte de la información a otros sectores. Esto ocurrió en sectores, que geológicamente se asocian a las rocas del Complejo de Nicoya, con pocos datos sobre la profundidad del nivel del agua y litología. Sin embargo, afortunadamente, la mayor proporción de estos terrenos con poca información se ubican en zonas con pendientes mayores al $40 \%$, recibiendo una protección ya definida en la Ley Forestal y, por lo tanto, no son susceptibles a ser desarrolladas. Por su parte, debido a la escaza información disponible en la zona, la metodología que se ajustó mejor a este estudio fue la del método GOD. De igual forma, se recomienda ejecutar, en la medida en que se genere más información, otros métodos de mayor precisión, como DRASTIC, propuesto por Aller et al. (1987), entre otros. Sin embargo, este método tiende a subestimar la vulnerabilidad de acuíferos fracturados (Foster et al. 2007), y otros métodos más complejos requieren de una mayor inversión en cuanto a la generación de insumos, como la tasa de percolación o de recarga acuífera. Además, según Foster et al. (2013), no necesariamente los métodos más complejos son los que generan mapas de vulnerabilidad más cercanos a la realidad.

En Costa Rica, los planes reguladores de ordenamiento territorial no toman en cuenta la amenaza y el riesgo a la contaminación y solamente incorporan la cartografía de vulnerabilidad hidrogeológica como una herramienta de gestión del recurso hídrico subterráneo. El mapa de riesgo de contaminación, al integrar la amenaza de contaminación y la vulnerabilidad de los acuíferos, permitiría identificar zonas de conflicto con el propósito de diseñar o corregir medidas regulatorias para la protección del agua subterránea. 


\section{Recomendaciones}

Tomando en cuenta el nivel de vulnerabilidad estimada, se generó una serie de recomendaciones de manejo y protección del suelo en la zona de estudio. Aun cuando se cuenta con información litológica limitada y se carece de pruebas de infiltración en el acuífero fracturado, se recomienda tomar varias medidas de protección de manera precautoria hasta que se conozca mejor la zona no saturada sobre el acuífero fracturado.

Es así como, en la llanura aluvial, la cual se clasificó como zona de Vulnerabilidad Hidrogeológica Moderada, se justifica la necesidad de la instalación de un sistema de alcantarillado sanitario, el cual cuente con un sistema de tratamiento, debido a la lixiviación potencial de sustancias contaminantes desde los tanques sépticos y letrinas. Se recomienda la introducción de esta medida de manera paulatina con apoyo del gobierno local, del AyA y del Consejo Cantonal de Coordinación Interinstitucional (CCCI) de Jacó. Así mismo, las plantas de tratamiento de aguas residuales mejoradas son obligatorias para las actividades hoteleras, turísticas e industriales de alto impacto, en concordancia con el concepto de armonía con el entorno, dirigido al ecoturismo y la sostenibilidad ambiental.

Por su parte, en el sector de vulnerabilidad moderada, es decir, sobre el acuífero detrítico, se recomienda que el cambio de uso de suelo de pasto y cultivos a urbano sea permitido, mientras se cumpla con las indicaciones sobre la disposición de aguas servidas mencionadas anteriormente. Además, se recomienda que los predios tengan un área mínima de $300 \mathrm{~m}^{2}$, con un área de construcción máxima del $50 \%$, para evitar la impermeabilización de los terrenos y favorecer la recarga del acuífero. De conservarse las zonas de cultivo en la zona del acuífero detrítico, se recomienda incentivar prácticas de manejo agrícola mejoradas, como el uso racional de agroquímicos, la agricultura orgánica, cosechas controladas para el descanso de los suelos y proyectos de reforestación. En esta zona, no se recomiendan los rellenos sanitarios, canteras y/o tajos.

Así mismo, en el sector de vulnerabilidad baja, sobreyaciendo el acuífero fracturado, las regulaciones pueden ser más flexibles y permisivas. En cuanto al desarrollo urbanístico, se recomiendan predios de un mínimo de $250 \mathrm{~m}^{2}$ y con un área de construcción máxima del $50 \%$, siempre y cuando se encuentren en terrenos que cuenten con una pendiente menor al $40 \%$. Al igual que en las zonas de Vulnerabilidad Moderada, la agricultura 
y el pastoreo deben de seguir prácticas de manejo de suelos adecuadas y en lo posible incentivar a la reforestación y a la agricultura orgánica. Las actividades turísticas y hoteleras deberían encaminarse al ecoturismo. Por último, se recomienda que sean permitidos: los rellenos sanitarios (con un diseño adecuado a las condiciones), los tajos, canteras y la industria con plantas de tratamientos mejoradas. Para los terrenos con una pendiente mayor al $40 \%$, se recomienda un uso de suelo forestal, el cual se ha designado como tal en la Ley Forestal de Costa Rica para, entre otras cosas, minimizar la erosión.

Finalmente, se recomienda implementar o seguir ejecutando otras herramientas de gestión integral del recurso hídrico: incentivos económicos para promover cambios hacia actividades con menor potencial contaminante en zonas con vulnerabilidad moderada y elevada; redes de monitoreo para la calidad del agua subterránea; e implementación de programas de concientización social derivados de un manejo de cuencas conjunto entre cantones y localidades, apoyados por universidades, las municipalidades, el gobierno y la empresa privada.

\section{Referencias}

Andreo, B., Vadillo, I., Carrasco, F., Neukum, C., Jiménez, P., Goldscheider, N., Hötzl, H., Vías, J.M., Pérez, I. y Göppert, N. (2004). Precisiones sobre el funcionamiento hidrodinámico y la vulnerabilidad a la contaminación del acuífero kárstico de la Sierra de Líbar (provincias de Málaga y Cádiz, Sur de España) a partir de un ensayo de trazadores. Revista de la Sociedad Geológica de España, 17(3-4): 187-197.

Albinet, M. y Margat, J. (1970) Cartographie de la vulnerabilite a la pollution des nappes d'eau souterraine [Contamination vulnerability mapping of groundwater]. Bulletin de la Bureau de Recherches Géologiques et Minieres. Segunda serie 3(4):13- 22.

Agudelo, C. (2005). Diagnóstico de las aguas subterráneas en las hojas Tárcoles, Herradura y Candelaria. Servicio Nacional de Aguas Subterráneas, Riego y Avenamiento. pp. 35. SENARA, San José. [Informe interno].

Aller, L., Bennett, T., Lehr, J., Petty, R. y Hackett, G. (1987). DRASTIC: A standardized system for evaluating geroundwater pollution potential 
using hydrogeologic settings. Environmental Protection Agency Report 600/2-87-035. Washington, D.C.

Arias, O. (2003). Redefinición de la Formación Tulín (Maastrichtiano-Eoceno Inferior) del Pacífico Central de Costa Rica. Revista Geológica de América Central, 28:47-68.

Arias, M. y Morera, S. (2002). Evaluación del acuífero de Jaco, cantón de Garabito, Provincia de Puntarenas. Servicio Nacional de Aguas Subterráneas, Riego y Avenamient. pp.15, SENARA, San José [Informe interno].

Barberá, J. A. y Andreo, B. (2015). Hydrogeological processes in a fluviokarstic area inferred from the analysis of natural hydrogeochemical tracers. The case study of eastern Serranía de Ronda (S Spain). Journal of Hydrology, 523: 500-514.

Barrantes, G. (2004). Disponibilidad del recurso hídrico y sus implicaciones para el desarrollo en Costa Rica. -25 págs. Undécimo Informe Estado de la Nación en Desarrollo Humano Sostenible, Programa Estado de la Nación. San José, Costa Rica. [Informe interno].

Baumgartner, P. (1984). El Complejo Ofiolitico de Nicoya (Costa Rica): Modelos estructurales analizados en función de las edades de los radiolarios (Calloviense a Santoniense). Citado en: Sprechmann, P. (1984). Estratigrafía del Complejo de Nicoya. En: Sprechmann, P. (Ed.). Manual de geología de Costa Rica, (115-123). Volúmen1: Estratigrafía. Editorial de la Universidad de Costa Rica. San José, Costa Rica.

Denyer, P. y Alvarado, G. (2007), Mapa Geológico de Costa Rica. - Escala 1: 400000, DGM-ECG-UCR, San José, Costa Rica.

Denyer, P., Montero, W. y Alvarado, G. (2003). Atlas tectónico de Costa Rica. - Escala 1: 500000, EUCR, San José, Costa Rica.

Estado de la Nación (2007). Decimotercer Informe Estado de la Nación en Desarrollo Humano Sostenible. Programa Estado de la Nación. San José, Costa Rica [Inf. interno]. pp. 437.

Foster, S. (1992). Unsustainable development and irrational exploitation of groundwater resources in developing nations an overview. $I A H$ Hydrogeology Selected Papers. Heise, Hannover. 3: 321-336.

Foster, S. e Hirata, R. (1991). Determinación del riesgo de contaminación de aguas subterráneas, una metodología basada en datos existentes 
Alicia Gómez-Cruz - Helga Madrigal-Solis - Christian Núñez-Solís

Hazel Calderón-Sánchez - Pablo Jiménez-Gavilán

Vulnerabilidad hidrogeológica en la zona costera de Jacó, Pacífico Central, Costa Rica.

[2a ed.]. - 90 págs. CEPIS, Centro Panamericano de Ingeniería Sanitaria y Ciencias del Ambiente, Lima, Perú.

Foster, S. e Hirata, R. (1988). Groundwater pollution risk assessment: a methodology using available data. WHO-PAHO-CEPIS, Lima.

Foster, S., Hirata, R. y Andreo, B. (2013). The aquifer pollution vulnerability concept: aid or impediment in promoting groundwater protection? Hydrogeology Journal,21, 1389-3922 p.

Foster, S., Hirata, R., Gomes, D., D'Elia, M. y Paris, M. (2007). Protección de la Calidad del Agua Subterránea. Banco Mundial, Washington, D.C. (pp. 115).

INEC (2012). Resultados generales. Censo Nacional de Población y VI de Vivienda 2011. Instituto Nacional de Estadística y Censos. San José, Costa Rica. Recuperado: www.inec.go.cr [Consulta 4/03/2015].

Kuypers, E.P. (1980). The geologic history of the Nicoya Ophiolite Complex, Costa rica, and its geotectonic significance. Amsterdam, The Netherlands. Tectonophysics 68(3/4): 233-255. Citado en: Sprechmann, P. (1984). Estratigrafía del Complejo de Nicoya. En: Peter Sprechmann (Ed.). Manual de geología de Costa Rica. Volúmen1: Estratigrafía. Editorial de la Universidad de Costa Rica. San José, Costa Rica. pp.320.

Llamas, M.R. y Custodio, E. (2003). Intensive use of groundwater: a new situation which demands proactive actions. En: Llamas, M.R., Custodio, E. (Eds.), Intensive Use of Groundwater, Challenges and Opportunities (13-34). A.A. Balkema Publishers. Lisse, Países Bajos..

Orozco, R. (2015). Propuesta de manejo de uso y cobertura de la tierra para la reducción del riesgo de contaminación del acuífero costero Jacó, Pacífico Central, Costa Rica. 56 p. (Tesis de Licenciatura Universidad Nacional), Heredia, Costa Rica.

PRCR (2007). Proyecto de Regularización Catastral y Registral. Cartografia digital y ortofoto. Escala 1:50000. Instituto Geográfico Nacional. San José, Costa Rica.

Reynolds, J. y Fraile, J. (2002): Presente y futuro de las aguas subterráneas en el Valle Central. En: Reynolds, J. (ed.): Manejo Integrado de Aguas Subterráneas. EUNED. San José, Costa Rica. pp. 313. 